\title{
On Attitudes Toward Terminology
}

\author{
Fahad Rashed Al-Mutairi, reply by Evelina Leivada
}

In response to "Misused Terms in Linguistics" (Vol. 5, No. 2).

To the editors:

The objective of Evelina Leivada's essay, as she makes clear, "is to attain a higher level of terminological clarity and coherence within the field of linguistics." There is no doubt that linguistics is in need of such an initiative, and Leivada's attempt at fulfilling it is in itself worthy of praise. But out of the ten key notions she focuses on, three seem highly problematic: Universal Grammar, faculty of language in the narrow sense, and grammaticality judgment. Leivada's discussion of these terms appear to be wanting in one respect or another.

Linguistics is a vast field of research, and generative grammar is one of its subfields. Chomskyan linguistics belongs to the latter and it is this branch of linguistics that the ten terms Leivada chose to focus on belong to. The title of her essay should really have been "Misused Terms in Chomskyan Linguistics." If not for the sake of accuracy, it would have at least been fair to many linguists who, sadly enough, may well be unfamiliar with, or simply uninterested in, Chomsky's work.

As to the objective of Leivada's essay, terminological clarity in linguistics-and for that matter, in any field of research-is certainly a must. Quarreling over the meaning of a term, aside from being tedious, is also a sign of a communication failure among practitioners in any field of knowledge, linguistics included. Leivada begins her essay by quoting Eörs Szathmáry, who complains-rightly so, in my view-that linguists "would rather share each other's toothbrush than each other's terminology." "This," as Leivada asserts, "is far from an isolated view." Now, if this is true, and I believe it is, it suggests that the problem goes deeper than simply fixing the semantic content of linguistic terms. It is not merely a problem of terminology; it is principally one of attitude.

Leivada's essay would have been much more satisfactory and useful to linguists had she taken the trouble to dig deeper than terminological clarity and consider linguists' attitudes toward both their own terminology and that of others. With respect to the former, it would be more instructive, for instance, to ask why theoretical terms come into fashion and fall out of it, and whether this has to do with empirical considerations or is simply a matter of caprice. I have in mind the so-called economy principles such as "procrastinate" and "greed," which had been given considerable attention in early stages of the minimalist program before they gradually fell out of use. As to the attitude which many linguists have toward each other's terminologies, it would be interesting to compare linguistics with physics in this respect. The latter is a field that takes terminology very seriously-so seriously, in fact, that a lack of consensus on a term's definition can suffice as a reason to jettison the term entirely. In a symposium on the physical concept of weight, one physicist complained that

the physics teaching community should at least agree on a definition of the word "weight." That we do not is very strong evidence to me that the word should simply never appear in the literature or textbooks. ${ }^{1}$

One could only wonder what consequences such a high standard would have for linguistics.

Leivada is right in saying that achieving terminological clarity in linguistics "may help improve its visibility in neighboring fields, such as other parts of psychology, biology, and neuroscience.” But on several occasions Leivada appears to take for granted that if Chomsky means $X$ and not $Y$, then this suffices for the clarification of a term. It may well be the case that the coiner of a term is the originator of the confusion associated with it. More importantly, rather than limiting herself to terminological clarification, Leivada would have offered an even better service to linguistics had she posed and attempted to answer the following question pertaining to the attitude of linguists toward their own terminology: Is it worth fixing the meaning of $\mathrm{X}$ without specifying the possible insights $\mathrm{X}$ would lead to?

In relation to the three problematic notion mentioned at the outset, consider first Universal Grammar (UG). Leivada asserts that, for Chomsky, "language universals" refer not "to properties that are universally attested to in 
all languages, but to computational properties of the mind that are universal because they arise from a species-universal innate ability." "A property P in a given language," she continues, "can reflect a universal computational principle even if $\mathrm{P}$ is not attested to in another language." On what grounds, then, can it be said that $P$ arises from a species-universal innate ability? The pre-minimalist strategy, as is well known, has always been that if $\mathrm{P}$ cannot possibly be derived from linguistic experience, then $\mathrm{P}$ must be innate, as opposed to learned. The answer to the above question is provided by the poverty-of-stimulus argument, according to which linguistic knowledge goes far beyond what the linguistic environment actually justifies. Now, since "innate" does not necessarily mean "genetically innate," the poverty-of-stimulus argument survived the transition to minimalism whose emphasis is on nongenetic nativism. In light of this, it is surprising to read Leivada saying that,

given that a complete list of all the UG principles has not been compiled, the possibility that these principles are, for the most part, not language-specific, but have cognitive, third factor roots, cannot be ruled out.

I fail to see the link between "a complete list of all the UG principles"-whatever that means-and their language-specificity. Given the minimalist bottom-up approach to UG, it would be appropriate to say that an almost empty list of UG principles or properties is an empirical target based on the assumption that third factor roots have something to do with language.

The second notion is the faculty of language in the narrow sense (FLN). Leivada asserts categorically that "FLN is not the same thing as UG. FLN is unique to humans and unique to language. UG does not have this character." The matter is not so straightforward. Although Leivada is certainly right in stating that confusing these two terms "is a mistake that many linguists have made," the confusion itself is something to be expected given the strong similarity between the meanings of the terms. More importantly, Chomsky himself seems to have contributed to this confusion. He defines UG as the "theory of the genetic endowment of" the language faculty-a definition that suggests an asymmetry between UG and FLN. ${ }^{2}$ The former concerns properties of language that are genetically determined, whereas the latter refers to properties of language that are genetically unique to it. ${ }^{3}$ In that sense, FLN is a special case of UG. But at another point in the same source, Chomsky defines UG as "the theory of the distinguishing features of human language," and again elsewhere he states that "UG consists of the mechanisms specific to [the language faculty]. ${ }^{4}$ From this perspective, there is no longer an asymmetry between UG and FLN, the two terms seem to be definitionally identical.

The third and final problematic notion is "grammaticality judgment." The distinction between "grammaticality" and "acceptability" is notorious for being a source of confusion, and Chomsky himself cannot be blamed for it. Indeed, as a careful reading of his early work confirms, he was both clear and consistent in his distinction between these two notions. Unfortunately, Leivada's attempt at clarifying what should have been clear all along has the opposite effect. Although she correctly defines grammaticality as conformity to the rules of a grammar, she nevertheless approves of labeling "grammaticality judgments" as a misnomer. "Speakers," she adds, "have intuitions only about their perception of linguistic stimuli." Speakers cannot judge a sentence's grammaticality but only its acceptability. But, as I have argued at length elsewhere, it is simply a fact that "native speakers can, in certain cases, judge a sentence's grammaticality," and it is precisely this fact that "makes linguistic intuition both (1) a convenient tool for the investigation of linguistic structure, and (2) an explanandum for which a theory is needed." ${ }^{5}$ This was indeed the strategy that Chomsky adopted in the mid-1950s.

\section{Fahad Rashed Al-Mutairi}

\section{Evelina Leivada replies:}

Finding common ground is a useful technique to aid dialogue. Unfortunately, when it comes to our views on the topic discussed in my essay and his reply, Fahad Rashed Al-Mutairi and I seem to disagree on almost everything. Having such disagreements is fine, especially when it comes to views and not facts. As Charles Darwin wrote in the epilogue of one of his most influential books,

False facts are highly injurious to the progress of science, for they often endure long; but false views, if supported by some evidence, do little harm, for everyone takes a salutary pleasure in proving their falseness [emphasis added]: and when this is done, one path towards error is closed and the road to truth is often at the same time opened. ${ }^{6}$

The first point on which I disagree with Al-Mutairi concerns the title I gave to my essay. Instead of "Misused Terms in Linguistics," he suggests I should have used "Misused Terms in Chomskyan Linguistics." I view "Chomskyan linguistics" as a term that deserves to be listed in a second list of misused, ambiguous, and confusing terms. If one uses a notion introduced by a specific scholar, this does not entail that she endorses all of this person's theses or that she wishes for her work to be embedded into an area of study that bears another person's name. This is not to deny the relevance of Noam Chomsky's work in the terms I discuss. He is indeed the proponent of many of them. This makes my essay a work that in part discusses terms used within the generative tradition, but this does not make it an essay in Chomskyan linguistics. More importantly, it is incorrect to attribute the misuse of some of the terms I discussed 
exclusively to the generative tradition, when there is ample evidence for such misuse in other linguistic frameworks.

I am also not convinced by the motivation that Al-Mutairi provides for the title he suggests. He writes,

The title of her essay should really have been "Misused Terms in Chomskyan Linguistics." If not for the sake of accuracy, it would have at least been fair to many linguists who, sadly enough, may well be unfamiliar with, or simply uninterested in, Chomsky's work.

My essay provided more than ten references to Chomsky's work for those who are unfamiliar with it. As for the uninterested ones, they are free to not read Chomsky, but my essay is not signed with Chomsky's name and should not be considered Chomsky's work, so this latter concern is unrelated to my discussion of misused terms in linguistics.

I agree with Al-Mutairi's suggestion that considering linguists' attitudes toward their terminology would provide a service to the field. This, however, requires a proper experimental study, while the aim of my essay was a theoretical review of the actual use of certain terms. I hope such an initiative will be undertaken in the near future.

In relation to terminological concerns, Al-Mutairi argues that in my essay I appear to take for granted that if Chomsky means $\mathrm{X}$ and not $\mathrm{Y}$ when he introduces a term, this suffices to clarify this term. I am not sure how he reached this conclusion, but this is not a claim I pursued. What I proposed instead is that when a scholar introduces a term $\mathrm{X}$ further defining it in a way $\mathrm{Y}$ and then another person uses the same term $X$ and defines it in a very different way $\mathrm{W}$, the term becomes polysemous, and communication problems may arise. Chomsky's endorsement of one definition instead of another obviously does not suffice for the clarification of a term; if it did, we would have no reason to talk about misused, inaccurate, ambiguous, or polysemous terms in (generative) linguistics.

Perhaps the greatest distance between Al-Mutairi's views and mine is found in the context of our understanding of Universal Grammar (UG). He argues "I fail to see the link between 'a complete list of all the UG principles'whatever that means-and their language-specificity." The link is robust and perhaps spelling it out in different words may be useful to other readers too. What are the UG principles? Imagine we compile all the candidates ever proposed in the literature. Then we can ask how many of these candidates have analogues in other cognitive domains or other species. Al-Mutairi is right that the list may be empty in light of the third factor approach, but this has not been demonstrated yet. If he thinks it has, it is surprising that he provides no references to this demonstration.

I would like to close this reply by drawing the readers' attention to Al-Mutairi's discussion of grammaticality and acceptability, because it a unique opportunity to show in practice how terminological confusion may arise. Al-Mutairi thinks I am wrong to have labeled the term grammaticality judgments a misnomer, because in previous work he has argued that native speakers can judge a sentence's grammaticality. This is an excellent example of how linguists use terms in different ways. Obviously, native naive participants can be asked if a sentence is grammatical. However, in linguistic tasks, participants are often instructed to disregard what the official books list as correct and provide an answer as to whether a sentence appears fine to them. In other words, the relevant tasks aim to tap into their idiolects; the tasks are not formal exams about how well participants remember the official grammar they learned at school. Therefore, native naive participants provide judgments of acceptability, and calling this response a grammaticality judgment is a misnomer. Al-Mutairi does not provide any arguments to refute this point; he simply argues that it is a fact that speakers have judgments about grammaticality, citing previous work where he makes the same claim, again without backing it up with arguments. To repeat, it is trivially true that if a person is asked whether a sentence is fine according to the official grammar books, they may be able to provide an answer, if they are literate and familiar with books of grammar. One may call this grammar exam a grammaticality judgment task, if one wishes to use this term in a way that is not typically used. However, even in this case, processing factors come into play and may lead participants to make an incorrect judgment, precisely because acceptability and grammaticality are separate notions. ${ }^{8}$

\section{Fahad Rashed Al-Mutairi is a PhD graduate from the University of Essex.}

\section{Evelina Leivada is a psycholinguist at the Universitat Rovira $i$ Virgili in Tarragona, Spain.}

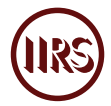

1. Ronald Brown, "Weight-Don't Use the Word at All," The Physics Teacher 37, no. 4 (1999): 241, doi:10.1119/1.880241.

2. Noam Chomsky, "On Phases," in Foundational Issues in Linguistic Theory: Essays in Honor of Jean-Roger Vergnaud, ed. Robert Freidin, Carlos Otero, and Maria Luisa Zubizarreta (Cambridge, MA: MIT Press, 2008), 134.

3. As an example, consider Chomsky's description of unbounded Merge as "not only a genetically determined property of language, but also unique to it." Chomsky, "Approaching UG from Below," in Interfaces + Recursion = Language?: Chomsky's Minimalism and the View from Syntax-Semantics, ed. Uli Sauerland and Hans-Martin Gärtner (Cambridge, MA: MIT Press, 2007), 5.

4. Chomsky, "Approaching UG from Below," 3. 
5. Fahad Rashed Al-Mutairi, The Minimalist Program: The Nature and Plausibility of Chomsky's Biolinguistics (Cambridge: Cambridge University Press, 2014), 13.

6. Charles Darwin, The Descent of Man (Princeton: Princeton University Press, 1871).

7. For example, the term "genetic endowment" or "blueprint" has been used by critics of the generative enterprise, even in works that recognize that Chomsky's original aim was to look to the overall biology of the organism as the source for grammar. See, for instance, Daniel L. Everett, "An Evaluation of Universal Grammar and the Phonological Mind," Frontiers in Psychology 7, no. 15 (2016): 1, doi:10.3389/fpsyg.2016.00015. It is worth highlighting that Everett argues that Chomsky "proposes that all languages are simply local manifestations of a biologically transmitted Universal Grammar" (p. 1), but to the best of my knowledge, Chomsky never claimed that Universal Grammar is transmitted. The process of genetic transmission refers to the passing of genetic material from parents to offspring. Language is neither a gene nor a chromosome, hence the use of the term transmission is wrong in that context. Given that Everett does not provide a reference to Chomsky's work when he attributes this point to him, I conclude that terms introduced within the generative tradition may be used incorrectly both inside and outside of it. This makes Al-Mutairi's suggestion for the title (i.e., "Misused Terms in Chomskyan Linguistics") a poor fit.

8. Evelina Leivada and Marit Westergaard, "Acceptable Ungrammatical Sentences, Unacceptable Grammatical Sentences, and the Role of the Cognitive Parser," Frontiers in Psychology 11, no. 363 (2020), doi:10.3389/fpsyg.2020.00364.

Published on September 28, 2020 